\title{
Investigating the Sporulation of Metarhizium anisopliae Formulated in Calcium Alginate in Soil ${ }^{+}$
}

\author{
Sudhan Shah *, Gavin Ash and Bree Wilson \\ Centre for Crop Health, Institute of Life Sciences and the Environment, University Of Southern Queensland, \\ Toowoomba 4350, Australia; gavin.ash@usq.edu.au (G.A.); bree.wilson@usq.edu.au (B.W.) \\ * Correspondence: sudhan.shah@usq.edu.au \\ + Presented at the Third International Tropical Agriculture Conference (TROPAG 2019), Brisbane, Australia, \\ 11-13 November 2019.
}

Published: 19 March 2020

Keywords: root crops; soil insects; entomopathogenic fungi; insect biological control; calcium alginate formulation

Wireworms have the potential to cause significant damage to sweetpotato, particularly late in the season. Whilst several insecticides are registered for use under minor permits, grower concern over the development of resistance has highlighted the need for alternative chemistry and management options. Entomopathogenic fungi like Metarhizium anisopliae have been shown to cause wireworm mortality in both laboratory and field conditions. Maintaining the abundance and persistence (i.e., resporulation) of fungi in soil is important for insect control; however, survivability declines in soils when the fungi is inundatively applied because the growth of fungi is limited and can also be parasitised or inhibited by other soil microbes. In this study, to mitigate this fungistasis, conidia of $M$. anisopliae were encapsulated in calcium-alginate granules with nutrients for the fungi in the form of corn starch or autoclaved baker's yeast or combination thereof. Combined corn starch and baker's yeast yielded the highest sporulation (granule denoted as $\mathrm{CAGMa}_{\mathrm{C}}+\mathrm{By}$ ). This study further investigated the infectivity of sporulated $\mathrm{CAGMa}_{\mathrm{C}} \mathrm{C}+\mathrm{By}$ for mealworms, and resporulation of CAGMa+Cs+By in field-collected soils that were either sterilised or not. Sporulated CAGMa+CS+By caused $>90 \%$ mealworm mortalities over 7 days bioassay. After 4 weeks incubation, resporulation of CAGMa+Cs+By in sterile soil was significantly greater (P value $>0.05$ ) compared to that observed in nonsterile soils (Figure 1). Further work is required to identify other additives that could be incorporated into granules to decrease the effect of fungistasis, to encourage entomopathogenic fungi to better establish in soil and to enhance the biological control of wireworm in sweetpotato. 

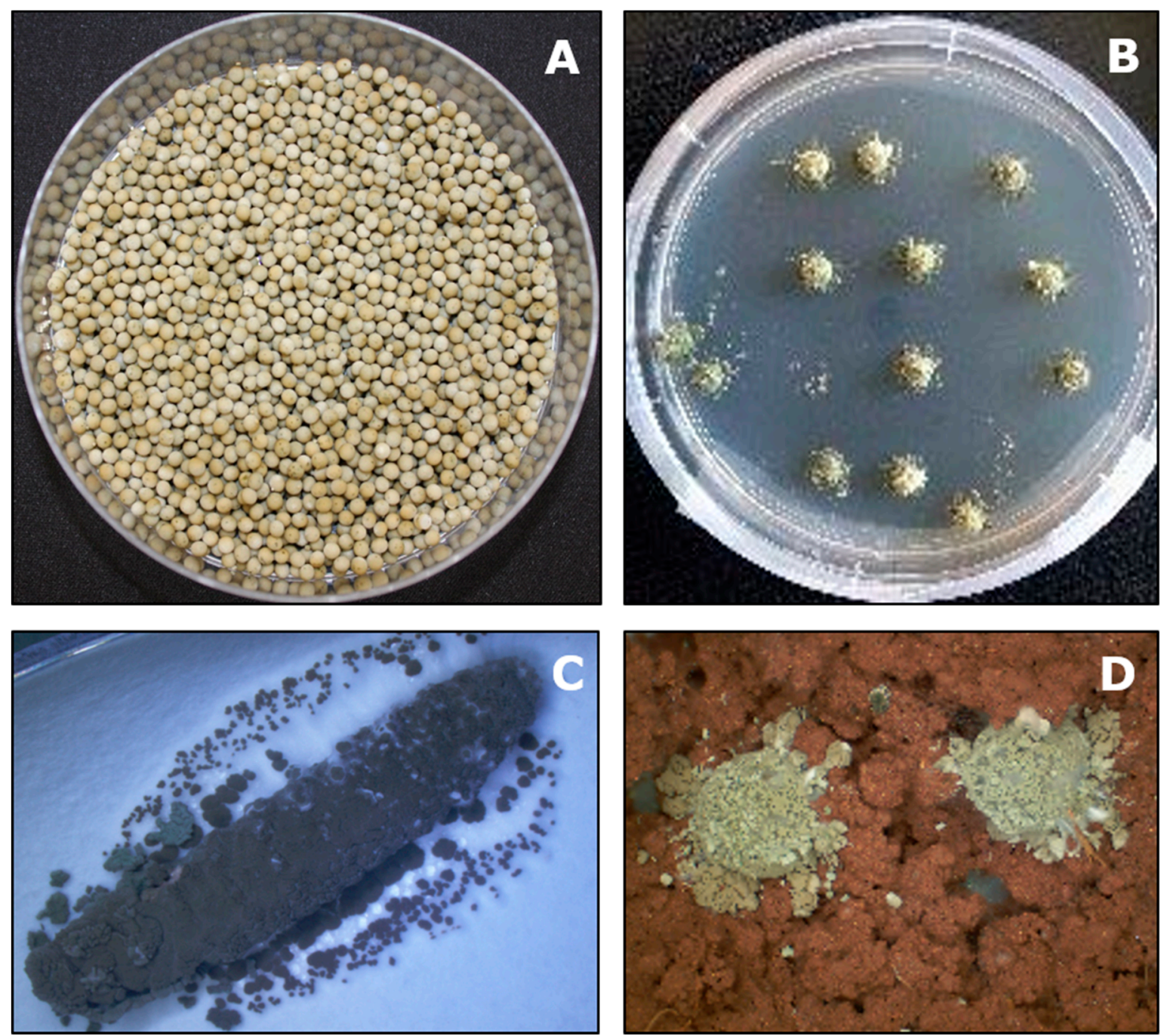

Figure 1. Metarhizium anisopliae formulated in calcium alginate granules (CAGMa+Cs+By) for soil insect control (A); the fungal granule $\mathrm{CAGMa}_{\mathrm{C}}+\mathrm{By}$ containing corn starch and autoclaved baker's yeast as the food source for $M$. anisopliae produced the optimal resporulation (B); a conidiated cadaver (mealworm) caused by the infection of resporulated CAGMa+Cs+By (C); and CAGMa+Cs+By resporulated in pasteurised soil following one month of incubation (D). (Photos source: Sudhan Shah).

Author Contributions: S.S. conducted the experiments and prepared the manuscript; B.W. prepared and edited the manuscript and provided supervision, and G.A. provided supervision.

Funding: This research was funded by the University of Southern Queensland under the scheme of postgraduate research scholarship and the Australian Centre for International Agricultural Research HORT/2014/083 project 'Developing improved crop protection options in support of intensification of sweet potato production in Papua New Guinea'.

Acknowledgments: We acknowledge the University of Southern Queensland for providing laboratory facilities; and the Crawford Fund for granting the scholarship to attend the conference.

Conflicts of Interest: The authors declare no conflict of interest.

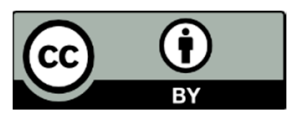

(c) 2020 by the authors. Licensee MDPI, Basel, Switzerland. This article is an open access article distributed under the terms and conditions of the Creative Commons Attribution (CC BY) license (http://creativecommons.org/licenses/by/4.0/). 